\title{
Learning from scratch a confidence measure
}

Matteo Poggi

http://vision.disi.unibo.it/ mpoggi

Stefano Mattoccia

http://vision.disi.unibo.it/ smatt
University of Bologna

Department of Computer Science and

Engineering (DISI)

Viale del Risorgimento 2

Bologna, Italy
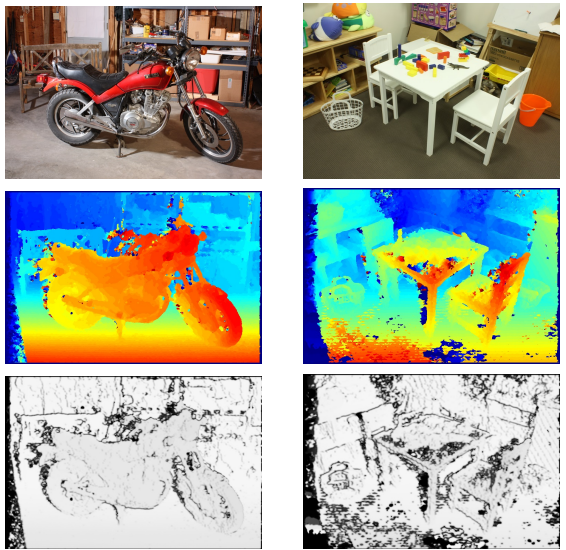

Figure 1: Reference image, disparity map and confidence computed by CCNN for Motorcycle and Playtable frames of the Middlebury 2014 training dataset.

In this paper, we propose a novel approach, referred to as Confidence Convolutional Neural Network $(\mathrm{CCNN})^{1}$ to predict the correctness of stereo matching by deploying a Convolutional Neural Network (CNN). In literature this is usually carried out by means of confidence measures [1] which encode the degree of reliability of the disparity assigned to each pixel by considering different cues: cost volume, reference image, disparity map and so on. Although some standalone measures are quite effective [1], recent works proved that combining a pool of them, within a machine learning framework, enables to significantly improve the overall effectiveness. In particular, Park \& Yoon [2] represents state-of-the-art in this field, obtaining the best results according to the Area Under the Curve (AUC) evaluation protocol defined by $\mathrm{Hu}$ and Mordohai [1].

This paper proposes the first method that

\footnotetext{
${ }^{1}$ The source code of $\mathrm{CCNN}$ and the trained network is available here: http://vision.disi.unibo.it/

\begin{tabular}{c|ccc} 
Dataset/Alg. & Opt. & Park\&Yoon & CCNN \\
\hline KITTI/BM & 0.137 & 0.179 & $\mathbf{0 . 1 7 5}$ \\
KITTI/SGM & 0.038 & 0.124 & $\mathbf{0 . 0 9 9}$ \\
Middl./BM & 0.093 & 0.114 & $\mathbf{0 . 1 0 7}$ \\
Middl./SGM & 0.042 & 0.093 & $\mathbf{0 . 0 7 4}$ \\
\hline
\end{tabular}

Table 1: Average AUC on KITTI 2015 and Middlebury 2014 training datasets with BM and SGM algorithms. The lower, the better. Values closer to optimum are in bold.

allows to obtain a confidence measure inferred from scratch by a CNN deploying as input cue only the disparity map computed by a stereo algorithm. This strategy makes our proposal suited even for out-of-the-box 3D sensors that typically do not provide the cues required by other methods.

For a fair comparison, we trained the proposed CCNN and Park \& Yoon [2] on KITTI 2012 (more than 6 million samples), using the Block Matching stereo algorithm (BM). This provides more than 6 million samples for training. Then, we evaluated CCNN and Park \& Yoon on KITTI 2015 training dataset processing the output of BM and Semi-Global Matching (SGM). We also cross-evaluated the two approaches, with BM and SGM stereo algorithms, on Middlebury 2014 training dataset. Table 1 reports average AUCs for CCNN and Park \& Yoon, computed on KITTI 2015 and Middlebury 2014, for BM and SGM, in order to assess their effectiveness. Observing the table, we can notice that our proposal always outperforms stateof-the-art.

[1] Xiaoyan Hu and Philippos Mordohai. A quantitative evaluation of confidence measures for stereo vision. IEEE Transactions on Pattern Analysis and Machine Intelligence (PAMI), pages 21212133, 2012.

[2] Min-Gyu Park and Kuk-Jin Yoon. Leveraging stereo matching with learning-based confidence measures. In The IEEE Conference on Computer Vision and Pattern Recognition (CVPR), June 2015. 